\title{
BioMedia for Entertainment
}

\author{
Ben Salem ${ }^{1, *}$, Adrian Cheok $^{2}$, and Adria Bassaganyes ${ }^{3}$ \\ ${ }^{1,3}$ Department of Industrial Design, Eindhoven University of Technology, The Netherlands \\ ${ }^{2}$ Mixed Reality Lab, National University of Singapore, Singapore \\ mail@bsalem.info, adriancheok@mixedrealitylab.org, \\ aketsocjo@hotmail.com, \\ www.bsalem.info
}

\begin{abstract}
In this paper we report on a novel form of media we call BioMedia. We introduce the concept and we explain its features. We then present two prototypes we have developed using BioMedia in entertainment.
\end{abstract}

Keywords: BioMedia, Multimedia, Empathy, Entertainment.

\section{Introduction}

BioMedia is a new media form that uses some property of living beings, in particular plants, as the medium for communication. Rather than use conventional media, say music, to render some information, BioMedia relies on health, shape, pigmentation or bioluminescence of living organism.

We would like to introduce the concept of BioMedia with our implementation of two entertaining systems. We focus on plants, in fact houseplants, as they have interesting features. Houseplants require continuous attention. Regular watering and soil checks are necessary for a houseplant to thrive and be healthy. Houseplants trigger empathy from their owner as s/he feel a duty to care for the plant. Plants can trigger emotions that go beyond what inanimate possessions can trigger. There are anecdotal evidences, when inquiring about ownership of houseplants, of an emotional and empathy link between owners and plants.

Houseplant form part of a house environment atmosphere and have a role in the overall perception one has of a room. We believe an entertainment value can be added if the plant is the embodiment of some information semantically coupled with users of that room.

BioMedia yield new opportunities for developing a new genre of media. The BioMedia form, content, and delivery are different from conventional media. First as living organism, houseplants cannot be controlled as accurately as electronic actuators. A time delay exists between stimuli and changes of conditions and the reaction from the plant. There is also a necessary minimal duration of stimuli for it to have an effect on the plant. It is difficult to assess with exactitude these parameters as they depend heavily on the individual plant size, light exposure, soil quality etc etc. In fact it is as if each plant has it own characteristics.

\footnotetext{
* Corresponding author.
} 


\subsection{How Does It Work}

Houseplants grow, change shape and react to various stimuli and to the characteristics of the soil they are living on. If taken good care of, houseplants will look healthy and beautiful. If neglected houseplants will wilt and eventually die. As such, there is an interesting opportunity in controlling the houseplant milieu, as it will be reflected in the plant state and appearance. In similar fashion, there is some potentially interesting effect in stimulating a houseplant, as it will result in some physical or chemical changes in the plant. The Concept of BioMedia originates from these opportunities. Described simply, BioMedia is about the control of a milieu or the delivery of certain stimuli as they influence some aspect of the plant.

Figure 1 illustrate the basic principle of BioMedia, a computer system via an actuator controls or influence some aspect of the plant milieu such as the moisture level, the orientation or the temperature. An alternative is for the computer system to influence the plant with particular stimuli, for example a light source.

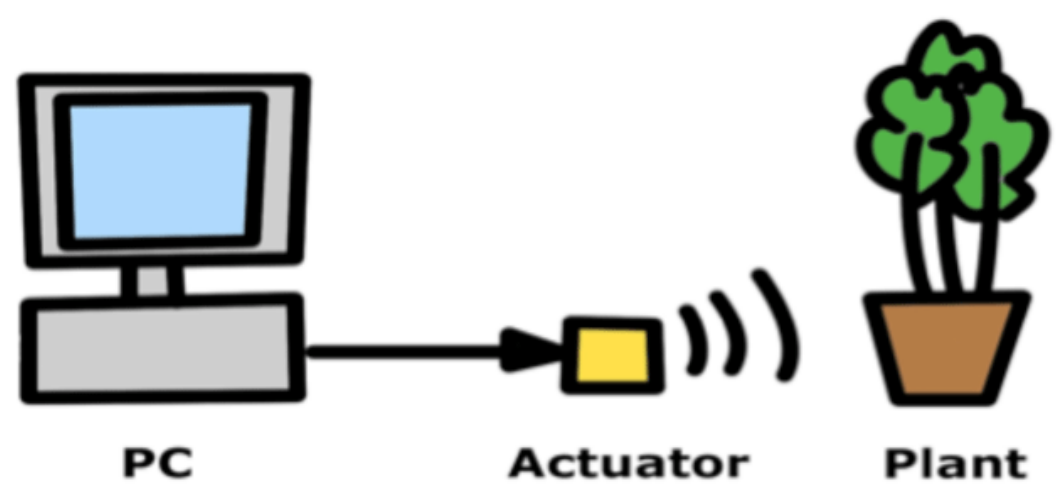

Fig. 1. Principle of BioMedia based on houseplant

In general a BioMedia system will display information over a certain amount of time as plant have adaptation latency. Depending on the plant and what aspects are changed, the adaptation can last anything between hours to months. The plant pigmentation change can take many months (3 months) to occur, as we have experienced with red cabbage (see next section). As a result there is a need to match the speed of change of the houseplant property with the bandwidth and the speed of change of the information displayed with the BioMedia system.

\subsection{Relevant Plant Features}

To use the plant as a display, we need to provide a control mechanism for one of it features. Several properties of the plant can be used such as health, growth, colour. Other properties can be used for our purpose, gravitropism, thermotropism, and phototropism.

By controlling the amount of water provided to a houseplant, one can control its rigidity and appearance. Wilting would result if a plant is not sufficiently watered. Severe wilting can also be triggered when carefully controlling the watering of the 
plant. There are some houseplants that are very resilient to water deficit such as cactus and other that are highly adaptive to irregular water availability such as the Fatsia Japonica. Plant colour can change depending on the soil conditions. Red Cabbage and Hydrangeas changes colour according to the soil $\mathrm{pH}$. Other feature of interest gravitropism, is the capability plants have to grow in opposite direction to gravity (i.e. upwards). If a plant is growing on an inclined ground, it will compensate for the slope and still grow vertically (as seen clearly with trees growing on hills). Thermotropism is the capability plants have to react to temperature changes or values. A Rhododendron for example curl its leaves when the temperature falls below $2^{\circ} \mathrm{C}$. Phototropism is the way plant will grow towards or away from the light source. Thigmotropism is the growth a plant will follow in reaction to a touch or contact. Usually thigmotropism occurs when plants will grow along a surface or around a standing element such as a tree trunk. Resurrection is the last feature we have looked at. It is the capability plants have to dry up and curl in and then blossom and reopen according to water availability (a good example of resurrecting plant is is the Rose of Jericho).

\subsection{Implementation in BioMedia}

Although it is possible to couple any plant feature with a BioMedia system, there are many limitations that need to be taken into account. The main one being the matching between the time duration needed for a plant feature to change and the speed of change of the information being displayed. This matching is necessary to ensure a strong coupling in the user's mind between the information and the plant feature.

We call the time duration for a plant feature change the feature latency, and it can range from seconds to years. At the fastest values range are the rapid plant movement, such as the Venus flytrap whose lobes snap shut when stimulated by a prey. Another

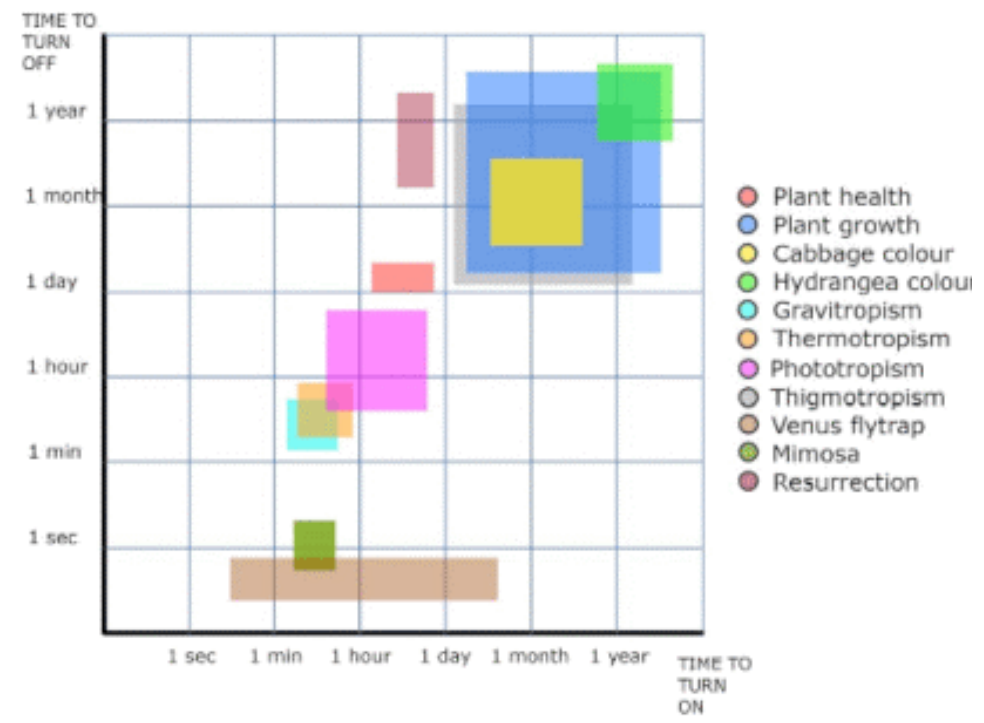

Fig. 2. Range of values for plant feature latencies 
feature with short latency is Mimosa (Mimosa Pudica L.) sensitivity to touch. The mimosa compound leaves fold inward and droop when touched and re-open within minutes. At the other end of the range are features such as plant growth and plant colour changes (such as the cabbage colour). These features have latency that range between months and years (see fig. 2).

Two plant features have attracted our attention, the plant colour and the plant health. We have selected Red Cabbage for plant colour changes and we have selected Fatsia Japonica for plant health changes.

\section{Related Work}

There are various projects using the principles of BioMedia. In this section we review only a limited number of these, selecting the closest ones to our proposal. Laughing Lily is a BioMedia system based on an artificial lily that has a carefully controlled blooming (see fig.3). The flower reacts to surrounding sounds. If nobody is talking the flower lets it petals droop [1].

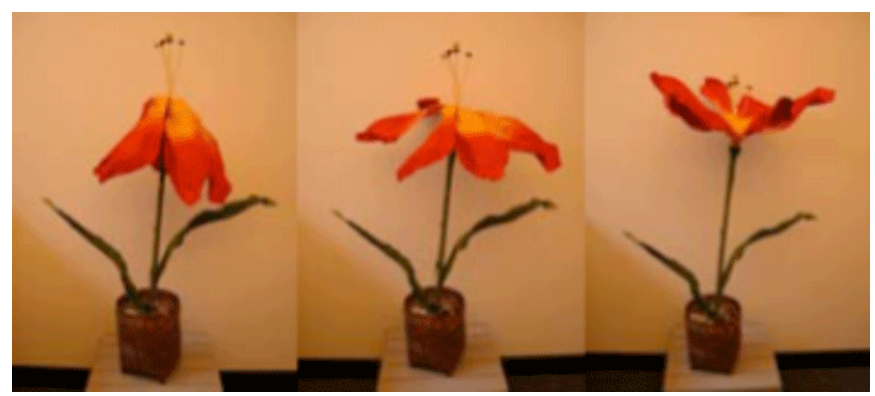

Fig. 3. Phototropism as used in the Infotropism project [1]

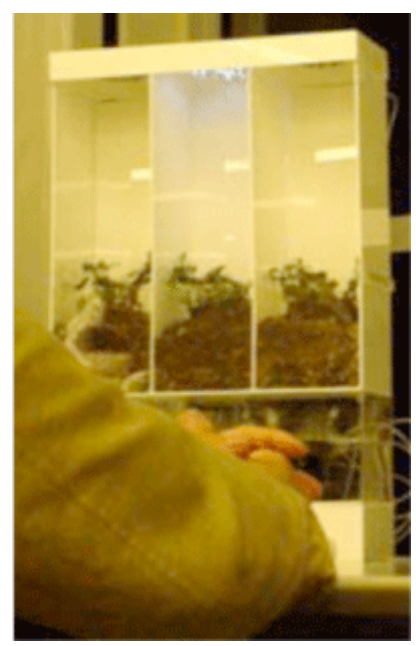

Fig. 4. Phototropism as used in the Infotropism project [2] 
Using real houseplants, PlantDisplay is a system where the growth of a plant is controlled by the amount of water given (see fig. 4). In the communication application, the watering of the plant is dependent on the number of telephone calls and emails one exchanges with friends [2].

With the infortropism project, it is the phototropic behaviour of the plants that is exploited to visualise the amount of recyclable rubbish in comparison to regular rubbish. Each time one of two rubbish bins is used, a light is activated. This light activation will gradually induce some plant growth towards it. So if there is more usage of a particular bin, this will result in a growth of plants towards its related light source (see fig. 5). There are also mechanical plants in a remote location that mimic the real plants [3].

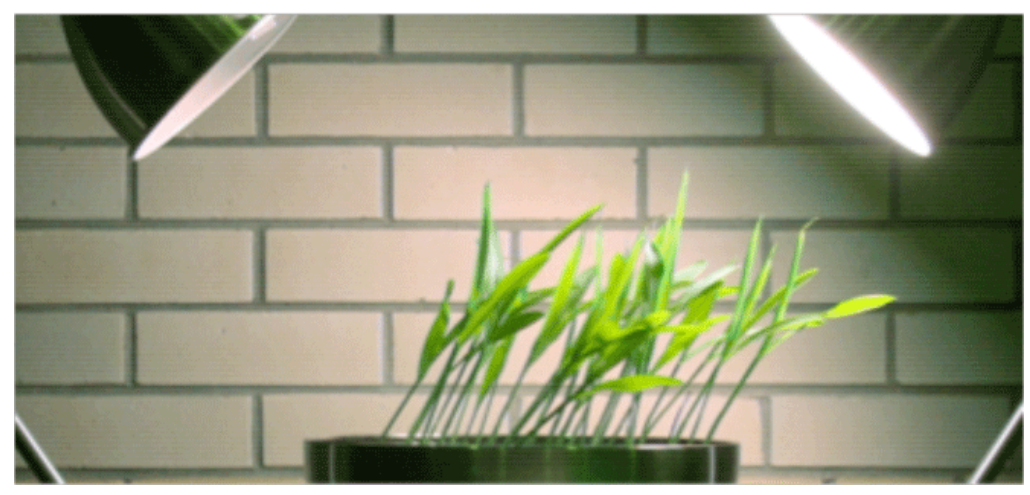

Fig. 5. Phototropism as used in the Infotropism project [3]

\section{BioMedia Applications}

The general direction that we have adopted was one of using BioMedia as part of a lifestyle display. In this scenario, various kind of information could be used. We have made a selection out of information pertaining to relationships, health, bad habits, money, and work. At the implementation level, we have investigated two applications using BioMedia. The first application we have developed with BioMedia is the display of a user's health information. The second application we have investigated is the display of lifestyle information.

In both cases we were interested in using the new media as an entertaining ambient system that connect people with houseplants via an empathy link. In general such system is made up of some sensors connected with the user, either directly to his/her body or indirectly through some personal information, for example a diary. The sensors collect some data related to the user. This data is processed into some information that is fed to the system and relate to the user lifestyle, health or personal habits. The information is then used to control some actuator that has an effect on the houseplant environment. These changes on the houseplant environment are reflected by some changes in the houseplant properties. These changes should not be to closely matching the actuator action; otherwise the control loop is too accurate. We need a 
loose accuracy as we are designing BioMedia as an entertainment system. As such we want to leave some scope for uncertainty and interpretation. An example that should work is the change of the soil acidity that has an effect on the houseplant pigmentation.

\section{Health Display}

In the health display we implement a BioMedia system coupling an indication of heart health with the colour of a plant, as outlined in fig. 6 .

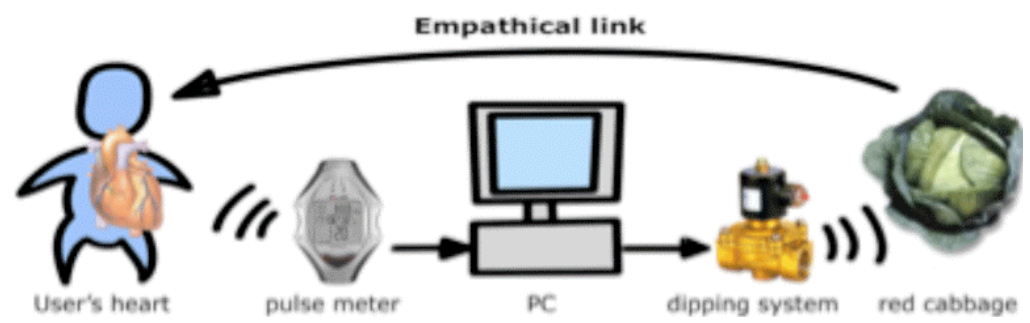

Fig. 6. Health information display system principle

The Red Cabbage change colour thanks to a natural pigment called anthocyanin. This pigment has the property of changing colour according to the plant milieu $\mathrm{pH}$ from green/blue for acid milieu to red for alkaline milieu. This explains why the plant is know as having different colour depending on the countryside region where it is growing (see fig. 7).

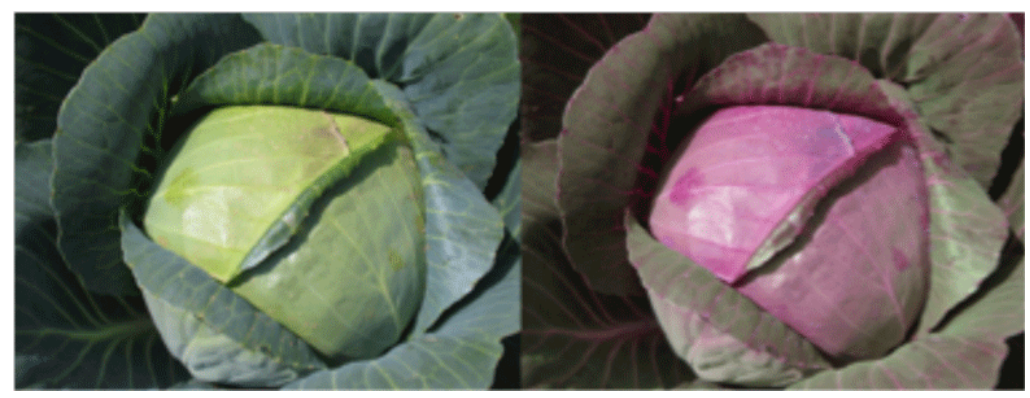

Fig. 7. Red cabbage with either a green (acid) or red (alkaline) natural pigmentation

In this system we have coupled the user's heart health with the cabbage pigmentation. If the user's hearth is healthy the cabbage will be pigmented green and if the user's hearth is not, the cabbage will be pigmented red. This is possible thanks to the Red Cabbage pigmentation dependence on soil acidity.

With our proposed system the user would have access to a non-intrusive display of his/her heart health (see fig. 8). The main application would be to warn users of the long-term negative effects of a bad heart health. It should be perceived as a complement to medical care, whereby it would indicate the need to improve heart health, based on a continuous monitoring of some heart parameters. 


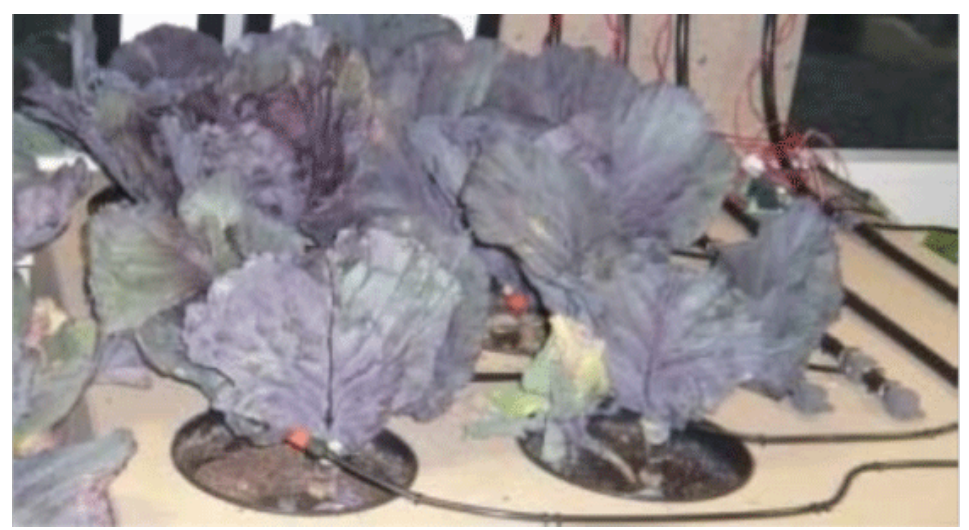

Fig. 8. Health display system with mature cabbage (note the dipping system)

\subsection{Input Data}

We have decided to focus on the heart as an indicator of the health condition of the user. Initial idea was to rely on the heart rate $(\mathrm{R})$ of the user. However $\mathrm{R}$ is not considered reliable enough as an indicator of heart condition. A better indicator is the heart rate variability (HRV). It is calculated as the fluctuation of time between heart beats. The HRV is the standard deviation of the $\mathrm{R}$ to $\mathrm{R}$ interval.

An average heart rate $\mathrm{R}$ would be around 60 beats per minute (bpm). This implies an HRV of $1.0 \mathrm{sec}$. However the heart is highly adaptive and the HRV of a healthy heart would fluctuate between $0.5 \mathrm{sec}$. to $2.0 \mathrm{sec}$. Typically a low HRV has been associated with a higher risk of heart problems and a high HRV with a healthy heart. Typically a HRV below $7 \mathrm{~ms}$ would be an indication of a rather unhealthy heart. An HRV above 100ms will be corresponding to a healthy heart (note that the HRV values do not follow a linear progression).

To calculate a user HRV we need to record $\mathrm{R}$ for a specific duration over a fixed period of time. In our case we have measured $\mathrm{R}$ of a user at night while sleeping. We have taken data samples over a 12 nights period. We discard the first hour of recording to give the user the opportunity to fall asleep; we then collect data over a two hours period. So we end up with data samples related to two hours over a 12 nights period.

Thanks to the control value ranges, we set up a system that waters the Red Cabbage with acid water (thus turning the plant green) if the HRV is above $70 \mathrm{~ms}$. The system will water the Red Cabbage with alkaline water (thus turning the plant red if the HRV is below $70 \mathrm{~ms}$.

\subsection{System Design}

For the purposes of our system we have created acidic water with a $\mathrm{pH} 3$ using Hydrochloric acid $(\mathrm{HCl})$ diluted in pure water. We have also created alkaline water with a pH 10 using Sodium Bicarbonate $\left(\mathrm{NaHCO}_{3}\right)$ diluter in pure water. Both water as well as pure water (for the control cabbage) were stored in three 20 liter jerrycans. The three jerrycans were located $3 \mathrm{mtrs}$ above the cabbages. With such arrangement, 
water was naturally flowing downwards into tubes. The water flow was adjusted thanks to electrical solenoid water valves. The valves are driven by a control board connected to a host PC (see fig. 9).

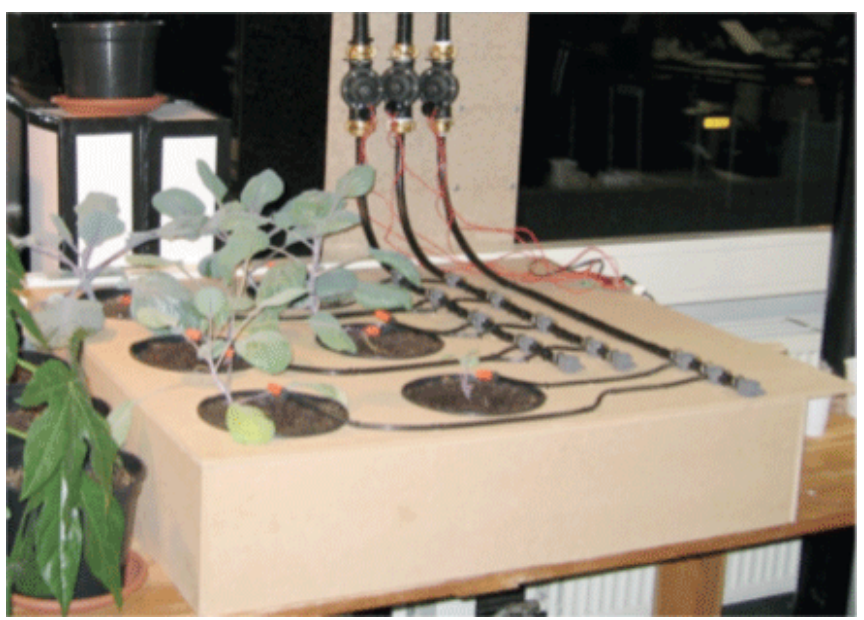

Fig. 9. Health display system (the valves are visible in the back of the installation and the dripping system is visible above each pot)

\subsection{Problems with the System}

The major problem we have encountered with this system is inherent to the principle of BioMedia. The use of plant as a display device requires some important changes to the plant appearance, in this case a significant change in the plant pigmentation which is a slow process that takes up to three months to start being effective. We have

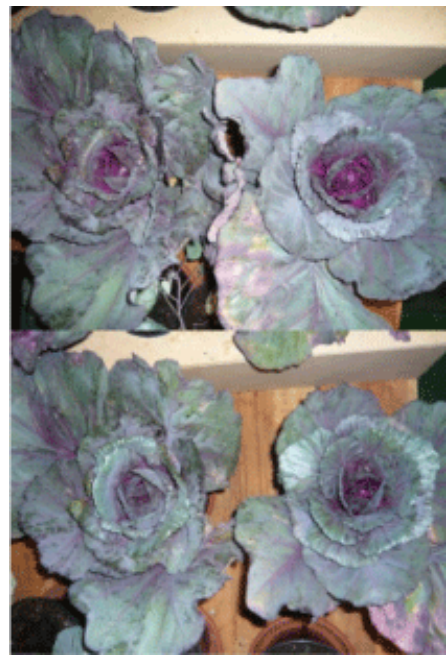

Fig. 10. Before (top) and after (bottom) watering with acid (left) and alkaline (right) water 
known that the feature latency of plant pigmentation is anything between one to several months. However there was no indication in the literature as to how much of a change will be visible. We have encountered the simple problem of the cabbage changing colour but not enough (as illustrated in figure 10). With hindsight we have realised that the acid and alkaline waters must make their way directly into the plant. The dipping display used was water the plant soil. Unfortunately, the plant soil acts as a $\mathrm{pH}$ buffer and reacts with the water used. A future version of the system will use a more direct delivery approach, probably based on an intravenous drip system directly connected to the plant main veins.

\section{Lifestyle Display}

It is a system coupling the smoking habits of staff using a smoking room in our campus with the health of a plant. We couple the usage of the smoking room with the amount of water fed to the plant. Users of the room are explicitly encouraged to discontinue smoking and can see the consequences of their actions via its negative effect on the plant health.

In this system we have used a sensor on the door of a smoking room in our campus to count the number of smokers who are using the room. The plant is watered in inverse relation to how frequently the room is used (see outline in fig. 11). If the room is empty the plant is watered, just enough to bring it back to a healthy state. If the room is being used the plant is left without water so as to wilt.

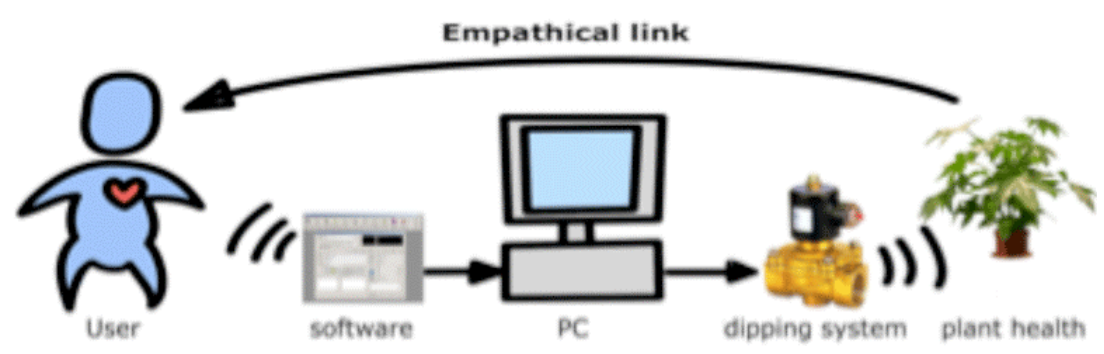

Fig. 11. Lifestyle display system principle

\subsection{Input Data}

We use a phidget to collect the data from the door sensor. This is fed to a C++ program running on a host PC. Every days, the program predicts the state of the plant by calculating the last 10days watering to assess the soil humidity and hence plant rigidity. Our program then calculates the number of smokers in the room for the last day. This number is used to calculate the desired plant rigidity and thus amount water. The amount of water is then used to control the system valve so as to open and close accordingly. 


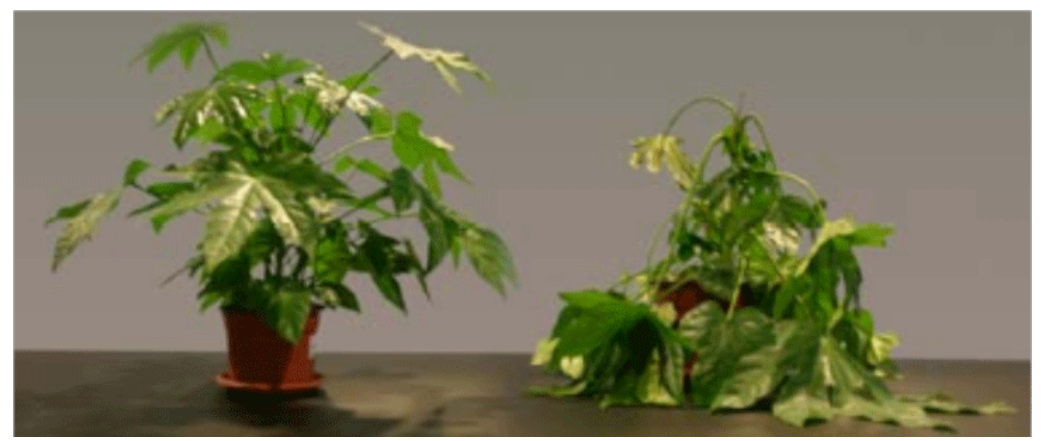

Fig. 12. Fatsia Japonica pronounced wilting without damage

\subsection{System Design}

Apart from a USB phidget and a host PC our system has a relay that drive an electrical valve. The watering system consists of one big plastic bottle, the electrical valve and a dipping tap. The water bottle is filled with a $\mathrm{pH}$ neutral water and positioned at the top of the installation (see fig. 13).

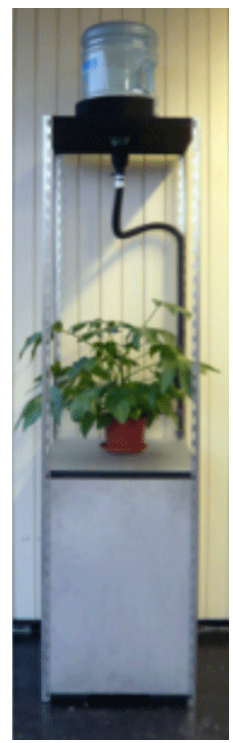

Fig. 13. Lifestyle display as a kiosk in a smoking room

It is important to implement a good control loop, whereby our system waters the plant in a precise enough manner so as to obtain a good effect. To do so we have carefully studied two plants and how they wilt and regain rigidity. We have used the results of this study to evaluate the exact amount of water to give to the plant and what effect this will have (details of this study are beyond the scope of this paper). 


\subsection{Problems with the System}

Surprisingly smokers were not too concerned with the health of the plant. We ran a trial by positioning the system in one of the smoking room in our campus, and we were expecting some reaction from the smokers. Instead, there has been some curiosity followed by indifference. One possible explanation is the denial of one's habit having an effect on the plant; instead believing that it is the others smoking habit that are negatively contributing to the plant health. Further investigations are obviously necessary to find out the truth.

\section{Conclusion}

Owners of houseplants are normally asked to provide proper light, temperature, water supply and a good soil to ensure a thriving and healthy plant. Thanks to BioMedia, they would also be asked to care for their health or to adopt a balanced and healthy lifestyle. It is obviously a drastic change of purpose for houseplants and an innovative development of media. Although we have only developed two prototypes we hope to continue this work with further systems and to investigate their usage, effect and affect on test subjects.

Thanks to the two early prototypes, we have determined that it is feasible to implement BioMedia systems the way we foresaw it. We also discovered a great potential for adaptation of BioMedia as an entertaining way to continuously monitor health and lifestyle. We hope to further this work and investigate various possible research directions.

Working on BioMedia has been very interesting as we have introduced in our research area things like plants, soil, water and seeds. We have combined electronic components and biological elements: A computer, a micro-controller, and a living plant. There were a lot of issues related to this interfacing. The digital and analogue world of electronic does not mix very well with the biological world of houseplants. One is controlled closely and in real-time. It requires explicit instructions to operate. The other is loosely controlled, with a latency that could last months. It only require various elements (water, soil...) and operates on its own rhythm and pre-programmed genetically behaviour. To make the connection between electronics and biology was an interesting challenge. In our case it is the flow of water that makes the connection. Interesting fact: water the most vital element for life is used to connect an electronic system with a biological organism.

\section{References}

1. Antifakos, S., Schiele, B.: LaughingLily: Using Flower as a Real-World Information Display. In: Dey, A.K., Schmidt, A., McCarthy, J.F. (eds.) UbiComp 2003. LNCS, vol. 2864, pp. 161-162. Springer, Heidelberg (2003)

2. Kuribayashi, S., Wakita, A.: PlatDisplay: Turning Houseplants into Ambient Displays. In: Proc. ACE 2006 (poster \#40). ACM, New York (2006)

3. Holstius, D., Kembel, J., Hurst, A., Wan, P., Forlizzi, J.: Infotropism: Living and Robotic Plants as Interactive Display. In: Proc. DIS 2004, pp. 215-221 (2004) 